\title{
Interplay of complex decay processes after argon $1 s$ ionization
}

\author{
R. Guillemin,${ }^{1,2}$ K. Jänkälä, ${ }^{3}$ B. Cunha de Miranda,,${ }^{1}$ T. Marin,,${ }^{1}$ L. Journel, ${ }^{1,2}$ T. Marchenko, ${ }^{1,2}$ O. Travnikova,,${ }^{1,2}$ \\ G. Goldsztejn, ${ }^{1}$ I. Ismail, ${ }^{1}$ R. Püttner, ${ }^{4}$ D. Céolin, ${ }^{2}$ B. Lassalle-Kaiser, ${ }^{2}$ M. N. Piancastelli, ${ }^{1,5}$ and M. Simon ${ }^{1,2}$ \\ ${ }^{1}$ Sorbonne Universités, UPMC Université Paris 06, CNRS, UMR 7614, Laboratoire de Chimie Physique Matière et Rayonnement, \\ F-75005, Paris, France \\ ${ }^{2}$ Synchrotron SOLEIL, l'Orme des Merisiers, Saint-Aubin, BP 48, 91192 Gif-sur-Yvette Cedex, France \\ ${ }^{3}$ Nano and Molecular Systems Research Unit, University of Oulu, P.O. Box 3000, 90014 Oulu, Finland \\ ${ }^{4}$ Fachbereich physik, Freie Universität Berlin, Arnimallee 14, D-14195 Berlin, Germany \\ ${ }^{5}$ Department of Physics and Astronomy, Uppsala University, P.O. Box 516, SE-751 20 Uppsala, Sweden
}

(Received 13 November 2017; published 23 January 2018)

\begin{abstract}
Complex decay pathways involving radiative and nonradiative relaxation after deep core-level ionization in argon are disentangled by a unique combination of several synchrotron radiation-based spectroscopic techniques. In particular, by comparing the results obtained from electron-ion coincidence, photon-ion coincidence, and $\mathrm{x}$-ray emission measurements, we are able to distinguish the final ionic states produced in the cascade decay involving $K \alpha$ and $K \beta$ radiative decay and final ionic states produced by nonradiative cascade decay. High-resolution Auger electron spectroscopy is then used as a complementary tool to identify the $L M M$ transitions contributing to the cascade decay. Ab initio calculations are performed to identify the electronic states involved in the $L M M$ decay.
\end{abstract}

DOI: 10.1103/PhysRevA.97.013418

\section{INTRODUCTION}

Absorption of an x-ray photon by an atom or a molecule promotes an electron from a deep shell to an empty orbital or into the ionization continuum. The resulting electronic state with an inner hole is highly unstable. When such inner vacancies are produced by hard $\mathrm{x}$ rays in heavy atoms, electronic relaxation can be accomplished in a variety of ways involving complex multistep cascades [1,2], radiative and nonradiative decays with shakeup and shakeoff of one or more electrons [3], as well as Coster-Kronig transitions, and more exotic pathways such as two-electron one-photon [4] and two-electron oneelectron decay [5]. The variety and abundance of possible decay channels can make the interpretation of Auger spectra quite difficult. Deep core-hole states have very short lifetimes, usually on the femtosecond time scale. Consequently, electronic state-lifetime interferences between various pathways leading to the same final states can further complicate the picture [6-11]. Moreover, fluorescence can play a role in the decay of the initial core-hole state, and the deeper the vacancy, the higher the probability of radiative decay compared to the Auger decay [12].

In this study, we report on a joint experimental and theoretical investigation of relaxation cascades after $1 s$ photoionization of argon. We show that, while different spectroscopic techniques can certainly give an accurate description of some aspects of such a complex process, our suitable unprecedented combination of several of those is crucial in gathering information on the interplay of all possible pathways, and therefore in deriving a complete picture of the overall phenomenon.

Argon is in industrial processes massively separated from air, inexpensive, safe to manipulate, and the $K$-shell ionization energy of $3200 \mathrm{eV}$ is easily accessible with an $\mathrm{x}$-ray tube or on modern synchrotron radiation sources. As such, argon has long been a model system to study phenomena associated with the creation of a deep inner-shell vacancy. An additional appealing feature of argon is that its closed-shell nature significantly simplifies theoretical treatment of the photoionization process. The $K$-shell ionization of argon was first studied by Carlson and Krause in 1965 using filtered radiation from an x-ray tube [1]. Following this pioneering study, more recent measurements were achieved using synchrotron radiation, with broadband excitation [13,14] and monochromatized radiation [15-17]. The most notable consequence of $1 s$ core ionization in argon is the production of highly charged ions up to 7+ [17]. The mechanism to create highly charged ions after single-photon absorption is in general understood in terms of cascade Auger decay. When an incident photon is absorbed and a $1 s$ electron is promoted to an empty orbital or to the continuum, the $1 s$ core hole created mainly decays by $K L L$ relaxation in which two electrons from the $L$ shell participate to the decay: one electron fills the hole and the other one is ejected into the continuum. The system is left in a highly unstable double core-hole state $L^{-2}$. This state will then again decay preferentially by Auger relaxation, involving the emission of more electrons. This cascading process leads to the production of ionic charges ranging from $1+$ to $7+, \mathrm{Ar}^{4+}$ being the main ion produced with a $46 \%$ probability [17].

The role played by fluorescence has been long recognized and has been studied by means of coincidence measurements between Auger electrons and fluorescence x-ray photons $[18,19]$. The relative weight of the radiative channel in $\mathrm{Ar}$ had been estimated between $8 \%$ and $14 \%[2,17,20]$. Radiative decay pathways branch into several subchannels [21]. $K \beta$ fluorescence (using Siegbahn notation, $K M$ using IUPAC notation), which is responsible for the formation of $\mathrm{Ar}^{+}$after $1 s$ ionization, leads to a state with a valence hole with no further energetically allowed decay. $K \alpha$ fluorescence $(K L)$, 
on the other hand, leads to a state with one $2 p$ hole that can further decay. $K \alpha$ fluorescence followed by $L M M$ Auger decay has been shown to be associated with the formation of $\mathrm{Ar}^{2+}$ [17]. Note that creation of this single $L^{-1}$ hole state following $1 s$ ionization can only be achieved by $K \alpha$ radiative decay, while $K L M$ Auger decay leads to a $L^{-1} M^{-1}$ two-hole state. Obviously, decay cascades involving fluorescence will lead to lower charge states and different Auger lines compared to purely nonradiative decay channels.

While both Auger electron and ion coincidences [13,14,22] and Auger electron and photon coincidences [18,19] have been used to study cascade decay following argon $1 s$ photoionization, no data are available on photon and ion coincidences. It is our aim in this paper to identify ionic final states and Auger lines associated with relaxation pathways involving radiative decay. To achieve this goal, we used coincidence measurements between photoelectron and ion, photon and ion, as well as fluorescence spectroscopy and Auger spectroscopy to clearly identify the contribution of radiative decays to the ion production following argon $1 s$ ionization, and to the $L M M$ Auger spectrum involved in the cascade decay of the initial core hole. Ab initio calculations of the intermediate and final electronic states were performed, and theoretical spectra are compared to experimental ones.

Our paper is organized as follows. Experimental and theoretical methods are described in Sec. II. In Sec. III, we compare the ion yields obtained in coincidence with photons and photoelectrons after direct $1 s$ ionization. Radiative relaxation is discussed in Sec. III A and the experimental and calculated LMM Auger spectra are discussed in Sec. III B. In Sec. IV, a short discussion of the results obtained after resonant excitation to the $4 p$ and $5 p$ Rydberg states is provided.

\section{METHODS}

\section{A. Experiment}

Photon-ion and photoelectron-ion coincidence measurements were performed on the LUCIA [23] and GALAXIES [24] beamlines at the French national synchrotron facility SOLEIL. The data were collected using a double momentum spectrometer [25]. In our experimental geometry, the photon beam crosses a cold supersonic jet of argon at a right angle, forming an interaction volume of approximately $0.1 \times 1 \times 2 \mathrm{~mm}^{3}$. In its original configuration, the setup is designed to measure electrons and ions in coincidence, using a static electric field to separate and accelerate charged particles towards two opposing time-of-flight spectrometers positioned perpendicularly to the photon beam and atomic jet. The time of flight and impact positions of the electrons and ions detected in coincidence are recorded, and used to derive the three components of the momentum vector of each particle. The efficiency of this recently developed setup has been validated by a series of successful experiments that provided a quantity of new results such as the electronic relaxation of core-ionized argon [17], post-collision interaction [26,27], and molecularframe photoelectron angular distributions [28,29]. In this study, we did not take advantage of the full momentum measurement capability of the apparatus. Only the time of flight of the ions was used to produce ion mass spectra. A $20 \mathrm{~V} \mathrm{~cm}^{-1}$ extraction

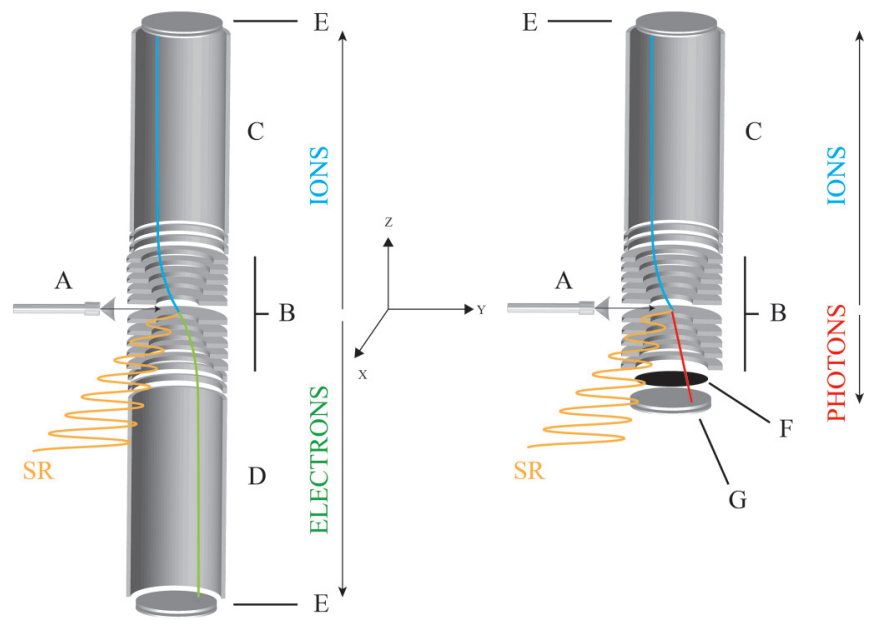

FIG. 1. Schematics of the coincidence setups. Left: electron-ion coincidence setup. A: molecular jet; B: electrostatic lenses; C: ion time-of-flight spectrometer; D: electron time-of-flight spectrometer; E: 80-mm microchannel plates; SR: synchrotron radiation. Right: photon-ion coincidence setup. F: aluminized-mylar filter; G: CsIcoated microchannel plates. See text for details.

electric field was used to collect all electrons and ions with a kinetic energy of $20 \mathrm{eV}$ or less within a $4 \pi$ solid angle. Measurements were performed at a photon energy $6 \mathrm{eV}$ above the $1 s$ ionization threshold (3206.26 eV [15]), and on top of the $1 s \rightarrow 4 p$ transition at $3203.5 \mathrm{eV}$, and $1 s \rightarrow 5 p$ transition at $3205.1 \mathrm{eV}$. The photon energy above threshold was chosen to minimize the effects of post-collision interaction and electron recapture on the ion rates $[17,30]$, while photoelectrons are still slow enough so they can be efficiently measured within a $4 \pi$ solid angle using a low extraction field.

To detect photons instead of electrons, the accelerating lens system was kept in place to preserve the homogeneity of the electric field for the ions, but one time-of-flight spectrometer was removed and replaced with a $0.5-\mu \mathrm{m}$-thick aluminizedmylar filter (see Fig. 1). A set of microchannel plates coated with cesium iodide was used for improved photon detection efficiency. Although the detection efficiency stays rather poor with this setup (estimated to 5\%), microchannel plate's signal is fast and particularly well adapted for coincidence measurements. Aluminized mylar provides an excellent filter blocking all charged particles and is essentially transparent to all photons with energies above $2000 \mathrm{eV}$ ( $>0.97 \%$ transmission above $2000 \mathrm{eV}$ and $0.99 \%$ transmission between 2950 and $3250 \mathrm{eV}$ ). On the opposite side, the setup remained unchanged. Coincidence events were digitalized with a time-to-digital converter triggered by the arrival of a photon on the coated channel plates and gated on the time of flight of the slowest ion, namely, $\mathrm{Ar}^{+}$.

The experimental measurements of high-resolution Auger spectra were carried out on the GALAXIES beamline, using the HAXPES end station dedicated to hard X-ray photoelectron spectroscopy [31]. Briefly, linearly polarized light is provided by a U20 undulator and monochromatized by a Si(111) double-crystal arrangement. Electrons are analyzed by a large acceptance angle EW4000 Scienta hemispherical analyzer, which lens axis is set parallel to the polarization axis of the synchrotron radiation. The unique capabilities of this apparatus 
for high-energy high-resolution spectroscopy allow revealing fine details of relaxation dynamics and have already provided a novel insight in the processes taking place after deep-core level ionization [32-35]. In this study, the electron spectrometer resolution was estimated to be $\sim 180 \mathrm{meV}$ at $100-\mathrm{eV}$ pass energy and the photon bandwidth delivered by the beamline is $350 \mathrm{meV}$ at $3200-\mathrm{eV}$ photon energy. Auger decay spectra were recorded on top of the $1 s \rightarrow 4 p$ and $1 s \rightarrow 5 p$ transitions, and $20 \mathrm{eV}$ above the ionization threshold, high enough to minimize the effect of post-collision interaction on the Auger lines [30], and low enough to neglect recoil effect such as measured on neon at high photon energy [32].

\section{B. Theoretical calculations}

The atomic-state calculations were carried out using the GRASP2K code [36,37] with the RELCI extension [38]. Standard GRASP2K calculations utilize the multiconfiguration DiracFock model where the atomic-state functions are formed as linear combinations of $j j$-coupled configuration state functions (CSFs) of the same total angular momentum and parity, and are optimized on the basis of the many-electron Dirac-Coulomb Hamiltonian. The CSFs are constructed from antisymmetrized products of a common set of orthonormal radial orbitals. Further relativistic corrections to the electron-electron interaction can be added later in a second step by diagonalizing the Dirac-Coulomb-Breit Hamiltonian matrix.

For simulating the ion yields and the measured Auger spectra, ionic states up to $\mathrm{Ar}^{+7}$ were calculated. Calculations of states were performed within the average level scheme where the radial orbitals of an ionic step are optimized to represent all states with equal weights. Cascades that started from the $\operatorname{Ar}\left(2 p^{-1}\right)$ states were first calculated using only the main configurations, meaning configurations constructed using the orbitals of the ground-state configuration $1 s^{2} 2 s^{2} 2 p^{6} 3 s^{2} 3 p^{6}$ of Ar plus $4 p$ or $5 p$ orbital in the $1 s \rightarrow n p$ excited cases. These calculations were, however, deemed unsuccessful because, for example, if the $\operatorname{Ar}\left(2 p^{-1} n l\right)$ states decay via Auger to $(3 s 3 p)^{-2} n l$ states only a single level is above $\mathrm{Ar}^{+3}$ states and no $\mathrm{Ar}^{+3}$ state is above the $\mathrm{Ar}^{+4}$ states. The calculations were therefore extended to include all configurations having single and double excitations to $3 d$ and $4 s$ orbitals. The number of configurations was limited by including only configurations that can directly interact with the main configurations via Dirac-Coulomb Hamiltonian. Cascades that started from the $\operatorname{Ar}\left(1 s^{-1}\right), \operatorname{Ar}\left(1 s^{-1} 4 p\right)$, or $\operatorname{Ar}\left(1 s^{-1} 5 p\right)$ states were calculated using only orbitals present in the ground-state configuration, plus $4 p$ or $5 p$ orbitals in the case of excited states. This limitation was necessary because, especially in the middle of the decay cascade, if more orbitals are included, the number of Auger transition matrix elements that need to be calculated rises to several millions. At the selected level, reproducing the full cascade required calculation of about 25000 transitions in the $\operatorname{Ar}\left(1 s^{-1}\right)$ case. In contrast, the total amount of Auger transitions required in the $\left(1 s^{-1} 4 p\right)$ and $\left(1 s^{-1} 5 p\right)$ cases was almost a million.

The Auger transition probabilities were calculated using the AUGER component of the RATIP package [39], that was modified to allow parallelized computation. The radiative transitions were modeled using the REOS program [40]. The AUGER
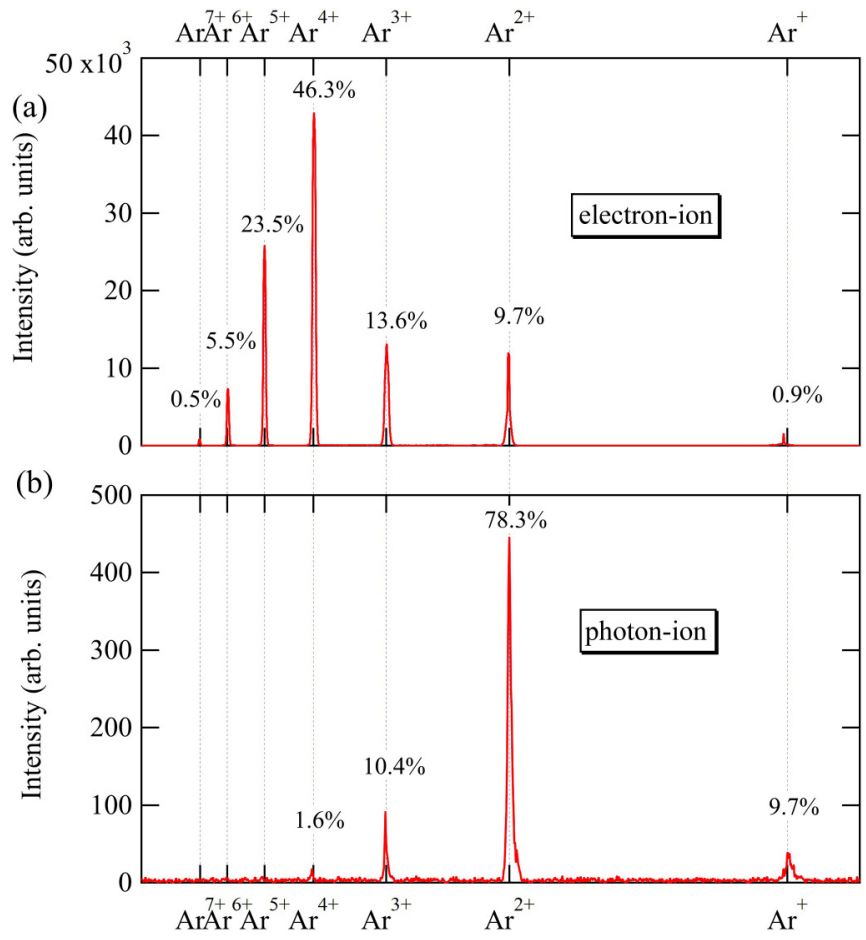

FIG. 2. Coincidence spectra measured $6 \mathrm{eV}$ above the argon $1 \mathrm{~s}$ ionization threshold: (a) photoelectron-ion coincidences, (b) photonion coincidences.

program can model only single Auger electron emission, meaning that higher-order processes such as direct double and triple Auger decays were not included in the calculations. This is not an issue when modeling normal Auger spectra because the excess energy is shared continuously in higher-order Auger processes. It, however, gives a small error to the simulation of ion yields.

\section{DIRECT IONIZATION}

Figure 2 shows the ion spectra obtained at a photon energy of $6 \mathrm{eV}$ above ionization threshold by coincidence measurements between ions and photoelectrons [Fig. 2(a)] and between ions and photons [Fig. 2(b)]. The measurement of all ions produced in coincidence with $1 s$ photoelectrons filters out ions produced by $\mathrm{x}$-ray absorption from higher electronic shells. This means that all ions measured in Fig. 2(a) are produced by the decay of a $1 s$ core hole, independently of the decay channel. Therefore, Fig. 2(a) also includes all the ions produced via decay channels that involve emission of a photon at one step of the cascade. By contrast [Fig. 2(b)], the coincidence spectrum shows only ions produced via decay involving at least one $K \alpha$ or $K \beta$ photon. Under our experimental conditions, the time it takes for the ion to travel from the interaction region to the detector is typically $12.5 \mu \mathrm{s}$ for $\mathrm{Ar}^{+}$and $4.7 \mu \mathrm{s}_{\text {for }} \mathrm{Ar}^{7+}$. Thus, the time of flight of the ion is much longer than the Auger cascade. As an example, the effective lifetime is $6.6 \mathrm{fs}$ for the production of $\mathrm{Ar}^{2+}$ [17]. Therefore, in our measurements, the coincidence between an electron or a photon is always with the final ionic product of the decay cascade. For both measurements, the ion branching ratios are given in the figure and reported 
TABLE I. Experimental and calculated ion branching ratios for photoelectron-ion and photon-ion coincidences. The photoelectronion branching ratios include all decay channels. The photon-ion branching ratios include only cascade decays involving either $K \alpha$ or $K \beta$ emission.

\begin{tabular}{lrrrrr}
\hline \hline & \multicolumn{2}{c}{ Photoelectron-ion } & & \multicolumn{2}{c}{ Photon-ion } \\
\cline { 2 - 3 } \cline { 5 - 6 } & \multicolumn{1}{c}{ Expt. } & Calc. & & Expt. & Calc. \\
\hline $\mathrm{Ar}^{+}$ & $0.9 \pm 0.1 \%$ & 1.21 & & $9.7 \pm 0.2 \%$ & 10.40 \\
$\mathrm{Ar}^{2+}$ & $9.7 \pm 0.1 \%$ & 10.66 & & $78.3 \pm 0.6 \%$ & 76.80 \\
$\mathrm{Ar}^{3+}$ & $13.6 \pm 0.1 \%$ & 11.23 & & $10.4 \pm 0.2 \%$ & 12.70 \\
$\mathrm{Ar}^{4+}$ & $46.3 \pm 0.2 \%$ & 53.49 & & $1.6 \pm 0.1 \%$ & 0.12 \\
$\mathrm{Ar}^{5+}$ & $23.5 \pm 0.1 \%$ & 17.12 & & & \\
$\mathrm{Ar}^{6+}$ & $5.5 \pm 0.1 \%$ & 6.29 & & \\
$\mathrm{Ar}^{7+}$ & $0.5 \pm 0.1 \%$ & 0.01 & & \\
\hline \hline
\end{tabular}

in Table I. Comparison of the two spectra shows as can be expected that ion production associated with radiative decay leads to lower charge states. Whereas the main ion produced after core $1 s$ ionization in argon is $\mathrm{Ar}^{4+}$, with a branching ratio of $46.3 \%$, ion production via radiative decay leads mainly to $\mathrm{Ar}^{2+}$, with a branching ratio of $78.3 \%$. Our calculated branching ratios, also summarized in Table I, are in good agreement with the measured branching ratios, except for the higher charges which are found with a much lower probability in the calculations in both cases. This discrepancy essentially comes from the difficulty to account for all the electronic states involved in complex decay cascades since calculations are intrinsically limited by the size of the configuration sets used to keep the calculation time reasonable. In addition, higher-order processes such as double and triple Auger decay were not included in the simulations.

\section{A. Radiative decay}

During $1 s$ ionization, a photoelectron is emitted to the continuum leaving a singly charged ion with a $1 s$ vacancy that we denote as $\operatorname{Ar}^{+*}\left(1 s^{-1}\right)$. Two radiative channels are available to the system, namely, $K \beta$ and $K \alpha$ decays. Using ion recoil analysis and post-collision distortion of the photoelectron lines, we already gave in two previous publications a tentative interpretation of the decay channels for the lower charge states $[17,26]$. Notably, we estimated that radiative decay represents $10.6 \%$ of all decay channels after argon $1 s$ core ionization [17]. This value comes from the observed branching ratios of $0.9 \%$ of $\mathrm{Ar}^{+}$and $9.7 \%$ of $\mathrm{Ar}^{2+}$. Indeed, these two ions can only be produced above threshold via decay channels involving radiative decay, with the exception of a weak $K M M$ Auger decay in the case of $\mathrm{Ar}^{2+}$. In $K \beta$ decay, a valence electron fills the $1 s$ decay and no further electronic decay is energetically possible (further neutral decay can involve the emission of a low-energy photon). $K \beta$ decay therefore leads to the production of $\mathrm{Ar}^{+}$. This process can be written as

$$
\gamma+\operatorname{Ar} \rightarrow e_{\mathrm{ph}}+\operatorname{Ar}^{+*}\left(1 s^{-1}\right) \rightarrow e_{\mathrm{ph}}+\gamma_{K \beta}+\operatorname{Ar}^{+}\left(3 p^{-1}\right) .
$$

In $K \alpha$ decay, one electron from the $2 p$ shell fills the $1 s$ vacancy, leaving a singly charged ion with a $2 p$ vacancy,

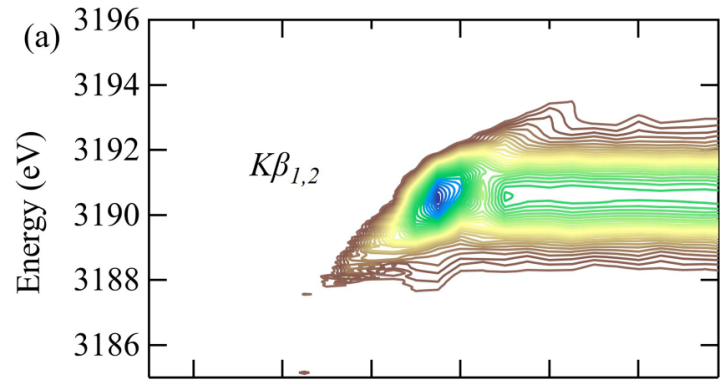

(b)
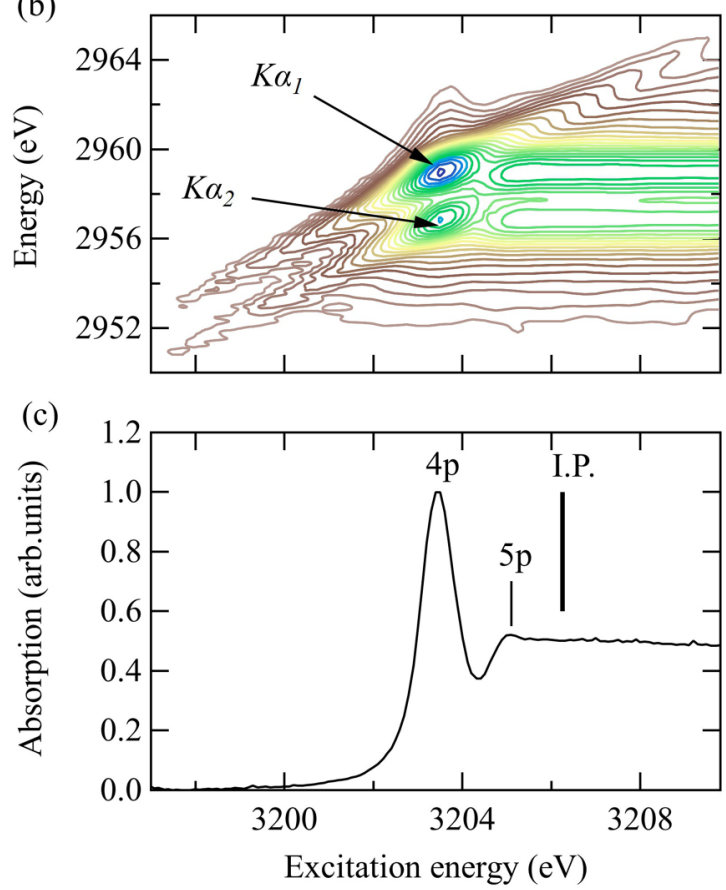

FIG. 3. 2D fluorescence map of argon around the $1 s$ ionization threshold. (a) $K \beta$ emission and (b) $K \alpha$ emission. Both maps show the emission intensity and emission energy as a function of excitation energy. (c) Corresponding absorption spectrum. See [21] for details. The positions of the $4 p$ and $5 p$ resonances, and the ionization threshold (I.P.) are indicated by vertical lines.

$\operatorname{Ar}^{+*}\left(2 p^{-1}\right)$, that can undergo further decay. Based on the recoil of the associated ion, we were able to identify the second step of this cascade as the nonradiative decay of the $2 p$ hole via emission of a $L M M$ Auger electron [17]:

$$
\begin{aligned}
\gamma+\mathrm{Ar} & \rightarrow e_{\mathrm{ph}}+\operatorname{Ar}^{+*}\left(1 s^{-1}\right) \\
& \rightarrow e_{\mathrm{ph}}+\gamma_{K \alpha}+\operatorname{Ar}^{+*}\left(2 p^{-1}\right) \\
& \rightarrow e_{\mathrm{ph}}+\gamma_{K \alpha}+e_{L M M}+\operatorname{Ar}^{2+}\left(3 p^{-2}\right) .
\end{aligned}
$$

Figure 3 shows the recorded $K \beta$ and $K \alpha$ radiative decay in argon following excitation around the $1 s$ ionization threshold. Data are taken from a previous publication [21], and are shown as two-dimensional (2D) maps as a function of excitation energy. $K \beta$ decay is identified in Fig. 3(a) as corresponding to the removal of $3 p$ electron. The two spin-orbit $1 s^{-1} \rightarrow 3 p_{3 / 2}^{-1}$ and $1 s^{-1} \rightarrow 3 p_{1 / 2}^{-1}$ components are separated by only $0.2 \mathrm{eV}$ and merge into a single line in the moderated-resolution spectrum. The two emission lines observed in $K \alpha$ decay correspond to 
$1 s^{-1} \rightarrow 2 p_{3 / 2}^{-1}$ and $1 s^{-1} \rightarrow 2 p_{1 / 2}^{-1}$ transitions separated by 2.2 $\mathrm{eV}$ due to spin-orbit interaction. They are respectively labeled $K \alpha_{1}$ and $K \alpha_{2}$ in Fig. 3(b). Figure 3(c) shows the absorption spectrum of argon around the $1 s$ ionization threshold and the position of the $1 s \rightarrow 4 p$ and $1 s \rightarrow 5 p$ resonances below threshold.

As discussed above, in process $1, K \beta$ decay leads solely to the production of the singly charged ion, and $\gamma_{K \beta}$ is the fluorescence photon detected in coincidence with $\mathrm{Ar}^{+}$in Fig. 2(b). This channel has a measured branching ratio of $9.7 \%$ of all ions associated with a radiative decay. It should be noted that $K \alpha$ decay to a $2 p^{-1}$ core-hole state followed by a secondary radiative decay to a singly charged $3 s^{-1}$ state also lead to the creation of $\mathrm{Ar}^{+}$but is at least two orders of magnitudes lower than $K \beta$ decay [20,41]. In process $2, K \alpha$ decay leads to the production of the doubly charged ion, and $\gamma_{K \alpha}$ is the fluorescence photon detected in coincidence with $\mathrm{Ar}^{2+}$ in Fig. 2(b), and $e_{L M M}$ is the second-step Auger electron. Although dominant, this channel is not the only pathway leading to the formation of the doubly charged ion. $\mathrm{Ar}^{2+}$ can also be produced through $K M M$ nonradiative decay $[22,26]$, which contributes to the partial yield in Fig. 2(a). Because $K \alpha$ decay produces an intermediate state with a single $2 p$ hole, the subsequent decay channels should be identical to the decay observed after $L$-shell ionization. Notably, in process 2 , we have written a final ionic state with two holes in the $3 p$ outer shell, $\mathrm{Ar}^{2+}\left(3 p^{-2}\right)$. To account for the production of $\mathrm{Ar}^{3+}$ and $\mathrm{Ar}^{4+}$ in Fig. 2(b), more complex pathways have to be included.

Previous ion yield measurements indicate a probability of forming $\mathrm{Ar}^{3+}$ between $10 \%$ [42,43] and $13.4 \%$ [44]. These values are in good agreement with the experimental value of $10.4 \%$ reported in Table I. The formation of $\mathrm{Ar}^{3+}$ requires a double Auger decay that may include cascade Auger decays and direct double decays. Double Auger decay after $L$-shell ionization in argon was first suggested by the observation of a large energy continuum in the recorded Auger spectrum [45]. The identification of $\mathrm{Ar}^{3+}$ states with $\left(3 p^{3}\right)$ and $\left(3 s^{1} 3 p^{4}\right)$ electronic configurations was later made possible using electronelectron coincidences [46], leading to a branching ratio for the triply charged states of $13 \%$. Using multiple electron coincidence measurements, Lablanquie et al. [47] studied in details the Auger decay of the $2 p^{-1}$ hole in argon showing that double Auger decay leads to the formation of $\mathrm{Ar}^{3+}$ and that $\left(3 p^{3}\right)^{4} S,{ }^{2} D$, and ${ }^{2} P$ states states are predominantly created as soon as the double Auger threshold is reached, with smaller contributions of $\mathrm{Ar}^{3+}\left(3 s^{1} 3 p^{4}\right)$ states and satellite states of $\left(3 p^{2} n l\right)$ configurations. The $\left(3 p^{3}\right)$ states are reached by cascade Auger decay, with the emission of a fast Auger electron followed by the emission of a slow Auger electron, with an $\mathrm{Ar}^{2+}$ intermediate state of $3 p^{2} 3 d^{2}$ configuration. The $\left(3 s^{1} 3 p^{4}\right)$ states are only associated with direct double Auger with the simultaneous emission of two electrons. From this study, double Auger decay was estimated to account for at least $9.1 \%$ of all Auger decay after the creation of a $2 p$ hole. In their study, Lablanquie et al. $[47,48]$ also report the observation of triple Auger decay leading to the production of $\mathrm{Ar}^{4+}$ with $0.2 \%$ of probability. From photoelectron-ion coincidence spectroscopy, Brünken et al. [44] previously reported a probability of $0.3 \%$. These numbers are almost an order of magnitude lower than the experimental value $1.6 \%$ found in our study, and slightly higher than our calculated value of $0.12 \%$. In the present calculations, $\mathrm{Ar}^{3+}$ and $\mathrm{Ar}^{4+}$ final ionic states are reached via $3 d$ and $4 s$ singly and doubly excited states in the first and second steps of the Auger cascade. Inclusion of even higher excited states would increase the percentage slightly, but most likely not to the observed experimental value.

\section{B. $L_{2,3} M M$ Auger decay}

The branching ratios of the different Auger decay pathways available after $1 s$ ionization in argon were calculated long ago by Chen et al. [49]. $K L_{2,3} L_{2,3}$ decay dominates largely the decay channels with a $55.3 \%$ probability, followed by $K L_{1} L_{2,3}$ (22.1\%), $K L_{1} L_{1}$ (6.88\%), $K L_{2,3} M$ (11.4\%), and $K L_{1} M(3.6 \%)$. $L M M$ Auger electrons are emitted during the second step in a cascade decay process and follow either $K L L$ Auger decay, $K L M$ decay, or $K \alpha$ radiative decay that leads to the creation of a single-hole $2 p^{-1}$ state. The ion formation observed in coincidence with fluorescence photons in Fig. 2(b) is associated with cascade Auger following $K \alpha$ decay, and should appear in the $L_{2,3} M M$ spectrum.

Interestingly, in the present case branching ratios (BR) of the first step of the Auger cascade combined with a knowledge of the highest possible ionic state that can be reached by the individual branches give a fairly good qualitative prediction and understanding for the observed ion yield (IY) in Table I. $K L_{2,3} M$ path terminates to $\mathrm{Ar}^{3+}$ which has $11.4 \% \mathrm{BR}$ in comparison to $13.6 \%$ IY, $K L_{2,3} L_{2,3}$ path terminates to $\mathrm{Ar}^{+4}$ with $55.3 \% \mathrm{BR}$ in comparison to $46.3 \% \mathrm{IY}, K L_{1} L_{2,3}$ and $K L_{1} M$ terminate to $\mathrm{Ar}^{5+}$ with $25.7 \%$ BR in comparison to $23.5 \%$ IY, and $K L_{1} L_{1}$ terminates mainly to $\mathrm{Ar}^{6+}$ with a

(a)

(b)

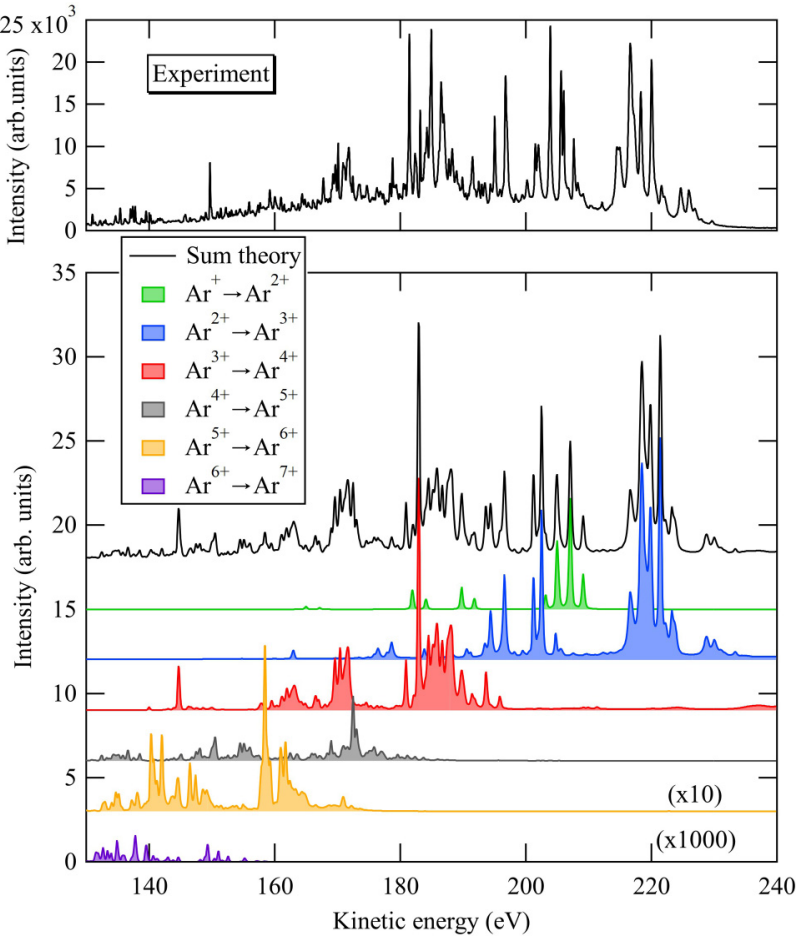

FIG. 4. $L_{2,3} M M$ Auger decay after core $1 s$ ionization. (a) Experimental spectrum measured $20 \mathrm{eV}$ above threshold. (b) Total calculated spectrum and partial $\mathrm{Ar}^{n+} \rightarrow \mathrm{Ar}^{m+}$ contributions. 
small possibility to reach $\mathrm{Ar}^{7+}$ which gives $6.88 \% \mathrm{BR}$ in comparison to $6 \%$ IY. The reason is that for every cascade the most intense decay channels remain open until the termination step. Therefore, the probability divided in the first step is almost completely transferred to the highest possible ionic state, except in the $\mathrm{Ar}^{7+}$ case. Obviously, deviations come from contributions of the fluorescence channels and earlier terminations.

Using the HAXPES setup at the GALAXIES beamline, we have measured the $L_{2,3} M M$ spectrum $20 \mathrm{eV}$ above the $1 s$ ionization threshold. Figure 4 shows the comparison of the experimental [Fig. 4(a)] and calculated $L M M$ Auger spectra [Fig. 4(b)]. This spectrum illustrates the complexity of the $L M M$ Auger spectrum after $1 s$ photoionization in argon where cascade processes dominate the Auger decay. We note that the spectrum measured here is a noncoincident spectrum contrary to the spectra recorded in coincidence with $K \alpha$ photons by Arp et al. $[18,19]$. Therefore, it includes all decay cascades involving radiative and nonradiative pathways. It is, however, possible to disentangle the different contributions with help of theory. Using broadband excitation (where all $K, L_{1}$, and $L_{2,3}$ shells are directly ionized), ion-Auger electron coincidence measurements, and relativistic calculations, von Busch

TABLE II. Electronic configurations of initial and final electronic states in the calculated $L_{2,3} M M$ Auger decay after argon $1 s$ core ionization.

\begin{tabular}{|c|c|c|c|}
\hline Transition & $\begin{array}{c}\text { Initial } \\
\text { configuration }\end{array}$ & $\begin{array}{c}\text { Final } \\
\text { configuration }\end{array}$ & $\begin{array}{c}\text { Kinetic } \\
\text { energy }(e V)\end{array}$ \\
\hline \multirow[t]{3}{*}{$\mathrm{Ar}^{+} \rightarrow \mathrm{Ar}^{2+}$} & $2 p^{5}$ & $3 p^{4}$ & $202-210$ \\
\hline & $2 p^{5}$ & $3 s^{1} 3 p^{5}$ & $180-194$ \\
\hline & $2 p^{5}$ & $3 s^{0}$ & $164-168$ \\
\hline \multirow[t]{6}{*}{$\mathrm{Ar}^{2+} \rightarrow \mathrm{Ar}^{3+}$} & $2 p^{4}$ & $2 p^{5} 3 p^{4}$ & $215-235$ \\
\hline & $2 p^{5} 3 p^{5}$ & $3 p^{3}$ & $198-208$ \\
\hline & $2 p^{4}$ & $2 p^{5} 3 s^{1} 3 p^{5}$ & 190-198 \\
\hline & $2 p^{5} 3 p^{5}$ & $3 s^{1} 3 p^{4}$ & $182-190$ \\
\hline & $2 p^{4}$ & $2 p^{5} 3 s^{0}$ & $172-182$ \\
\hline & $2 p^{5} 3 p^{5}$ & $3 s^{0} 3 p^{5}$ & $159-164$ \\
\hline \multirow[t]{6}{*}{$\mathrm{Ar}^{3+} \rightarrow \mathrm{Ar}^{4+}$} & $2 s^{1} 3 p^{4}$ & $3 s^{1} 3 p^{3}$ & $230-240$ \\
\hline & $2 s^{1} 3 p^{4}$ & $3 s^{0} 3 p^{4}$ & $215-230$ \\
\hline & $2 s^{1} 2 p^{5} 3 p^{5}$ & $2 s^{1} 3 p^{3}$ & $200-215$ \\
\hline & $2 p^{5} 3 p^{4}$ & $3 p^{2}$ & $178-197$ \\
\hline & $2 p^{5} 3 p^{4}$ & $3 s^{1} 3 p^{3}$ & $155-178$ \\
\hline & $2 p^{5} 3 p^{4}$ & $3 s^{0} 3 p^{4}$ & $139-153$ \\
\hline \multirow[t]{4}{*}{$\mathrm{Ar}^{4+} \rightarrow \mathrm{Ar}^{5+}$} & $2 p^{5} 3 s^{1} 3 p^{4}$ & $3 s^{1} 3 p^{2}$ & $168-190$ \\
\hline & $2 p^{5} 3 p^{3}$ & $3 s^{1} 3 p^{2}$ & $158-168$ \\
\hline & $2 p^{5} 3 s^{1} 3 p^{4}$ & $3 s^{0} 3 p^{3}$ & $140-158$ \\
\hline & $2 p^{5} 3 p^{3}$ & $3 s^{0} 3 p^{3}$ & $130-140$ \\
\hline \multirow[t]{5}{*}{$\mathrm{Ar}^{5+} \rightarrow \mathrm{Ar}^{6+}$} & $2 p^{5} 3 s^{1} 3 p^{3}$ & $3 s^{1} 3 p^{1}$ & $160-180$ \\
\hline & $2 p^{5} 3 p^{2}$ & $3 s^{2}$ & $157-160$ \\
\hline & $2 p^{5} 3 p^{2}$ & $3 s^{1} 3 p^{1}$ & $136-155$ \\
\hline & $2 p^{5} 3 s^{1} 3 p^{3}$ & $3 s^{0} 3 p^{2}$ & $136-155$ \\
\hline & $2 p^{5} 3 p^{2}$ & $3 s^{1} 3 p^{1}$ & $132-136$ \\
\hline $\mathrm{Ar}^{6+} \rightarrow \mathrm{Ar}^{7+}$ & $2 p^{5} 3 s^{1} 3 p^{2}$ & $3 s^{1} 3 p^{0}+3 s^{0} 3 p^{1}$ & $130-160$ \\
\hline
\end{tabular}

et al. [14] tentatively provided a complete assignment of the spectator satellites observed in the $L M M$ spectrum of argon corresponding to the transitions to the lower ionic states (up to $\mathrm{Ar}^{4+}$ ). The purpose of this paper is not to detail all the multitude of individual transitions calculated and resulting spectroscopic terms, therefore, we describe the transitions solely in terms of atomic configuration for the sake of simplicity and clarity, and we include all transitions up to $\mathrm{Ar}^{7+}$.

Our calculated decay spectrum shown in Fig. 4(b) includes Auger transitions between different ionic states that fall into the $120-240 \mathrm{eV}$ kinetic energy range. Both the experimental and calculated spectra are very similar to the $L_{2,3} M M$ spectra we obtained for $\mathrm{HCl}$ [50], and we can distinguish in the spectrum transitions we can associate to various decay process. To present our results in a form relevant to our discussion, we regroup the contributions of the many electronic states in terms of transitions between ionic states, i.e., for instance we group together all the transitions from a $\mathrm{Ar}^{1+}$ ionic state to $\mathrm{a}^{2+}$ ionic state. These groups represent the subspectra shown in Fig. 4(b) and they are shown in detail in Figs. 4 to 7. The partial $L_{2,3} M M$ Auger spectra obtained allow us to clarify the relaxation pathways leading to the ion production measured in Fig. 2. The transitions identified are detailed in Table II. However, it is important to recognize that not all ion production channels are associated with Auger transitions observed in this energy range.

\section{1. $\mathrm{Ar}^{+} \rightarrow \mathrm{Ar}^{2+}$ transitions}

The partial Auger spectrum associated with $\mathrm{Ar}^{+} \rightarrow \mathrm{Ar}^{2+}$ transitions corresponds to the well-known decay of the $2 p^{-1}$ core hole [51] and is displayed in Fig. 5. In the case of an initial $1 s$ ionization, these contributions to the $L M M$ Auger spectrum follow the radiative decay of the $1 s$ vacancy through

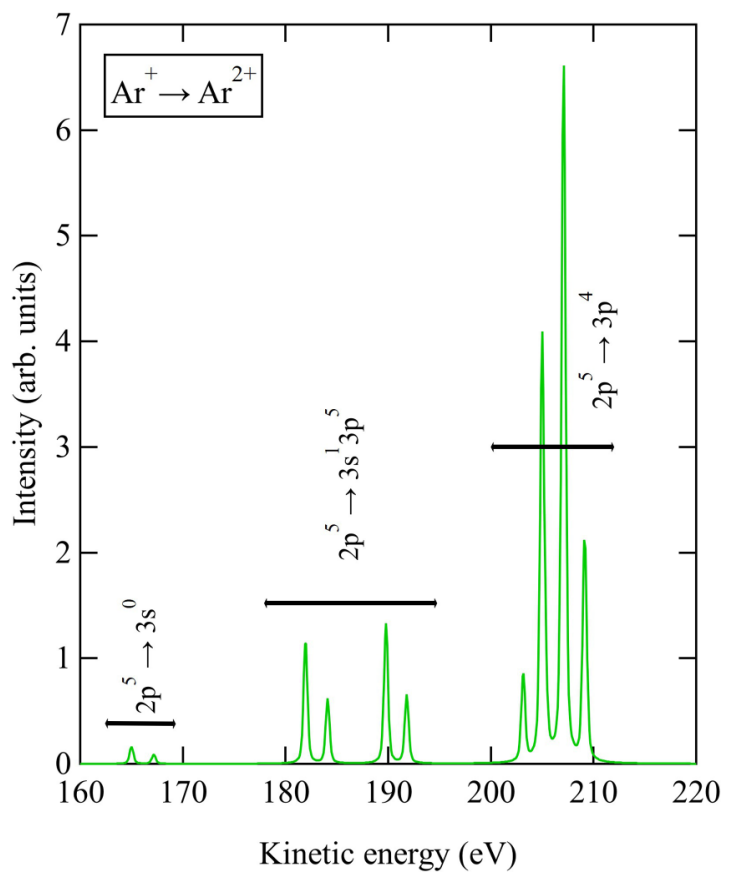

FIG. 5. Partial $\mathrm{Ar}^{1+} \rightarrow \mathrm{Ar}^{2+}$ contribution to the $L_{2,3} M M$ Auger decay after core $1 s$ ionization. 


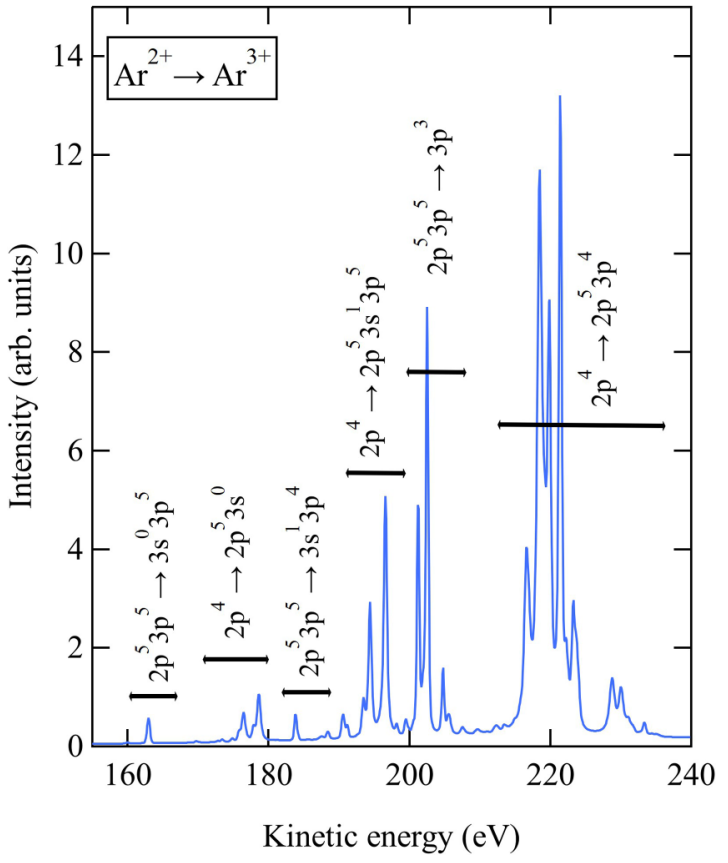

FIG. 6. Partial $\mathrm{Ar}^{2+} \rightarrow \mathrm{Ar}^{3+}$ contribution to the $L_{2,3} M M$ Auger decay after core $1 s$ ionization.

$K \alpha$ emission, and the creation of a single $2 p$ vacancy, as described above in process 2 . The filling of the $2 p$ vacancy by $L_{2,3} M_{2,3} M_{2,3}$ decay results in $2 p^{5} \rightarrow 3 p^{4}$ transitions, leaving the ion with two $3 p$ vacancies and leading to ${ }^{1} S_{0},{ }^{1} D_{2}$, and ${ }^{3} P_{0,1,2}$ final states. The $2.2-\mathrm{eV}$ spin-orbit splitting of the $2 p$ level into $2 p_{3 / 2}$ and $2 p_{1 / 2}$ core-hole states leads to the partial overlap of theses configurations and to the four peaks observed in the 202-210 eV kinetic energy region that was the particular focus of Arp et al. $[18,19]$ in their coincidence study. $L_{2,3} M_{1} M_{2,3}$ decay of the $2 p$ vacancy corresponds to $2 p^{5} \rightarrow 3 s^{1} 3 p^{5}$ transitions which lead to the creation of ${ }^{1} P_{1}$ and ${ }^{3} P_{0,1,2}$ final states in the $180-194 \mathrm{eV}$ kinetic energy region. Here, the spin-orbit splitting leads to the presence of two groups of two peaks, as observed in this energy region. The last group of peaks observed at lower kinetic energy, 164-168 $\mathrm{eV}$, corresponds to the $L_{2,3} M_{1} M_{1}$ decay of the $2 p$ vacancy, $2 p^{5} \rightarrow 3 s^{0}$, leading to two spin-orbit transitions to the ${ }^{1} S_{0}$ final state.

Lablanquie et al. [47] observed that the double Auger decay of the $2 p^{-1}$ core hole can also produce triply charged $\mathrm{Ar}^{3+}$ ions with $\left(3 p^{3}\right)$ configurations through a cascade process and $\left(3 s^{1} 3 p^{4}\right)$ configurations through direct double Auger decay.

\section{2. $\mathrm{Ar}^{2+} \rightarrow \mathrm{Ar}^{3+}$ transitions}

The partial Auger spectrum associated with $\mathrm{Ar}^{2+} \rightarrow \mathrm{Ar}^{3+}$ transitions is shown in Fig. 6. As previously noted by von Busch et al. [14], the majority (estimated to 87\%) of the initial $K L_{2,3} L_{2,3}$ transitions leads to a $2 p^{4}\left({ }^{1} D_{2}\right)$ configuration. Most of the transitions regrouped in the $\mathrm{Ar}^{2+} \rightarrow \mathrm{Ar}^{3+}$ curve in Fig. 6 correspond to the hypersatellites of the $L_{2,3} M M$ decay, i.e., the first step, $2 p^{-2} \rightarrow 2 p^{-1}$, in the sequential decay of the $2 p^{-2}$ double vacancy with an initial $2 p^{4}$ configuration created by $K L L$ decay. The resulting $2 p^{-1}$ vacancy undergoes further decay. One can not completely rule out radiative decay

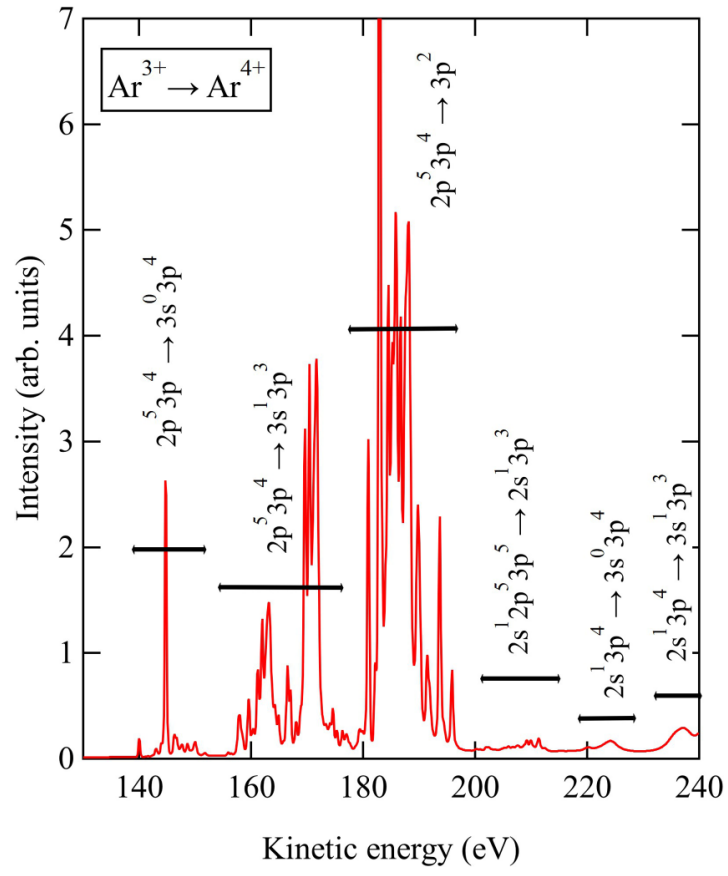

FIG. 7. Partial $\mathrm{Ar}^{3+} \rightarrow \mathrm{Ar}^{4+}$ contribution to the $L_{2,3} M M$ Auger decay after core $1 s$ ionization.

of the $2 p^{-1}$, however, fluorescence above the $2 p$ threshold is expected to be extremely weak [52]. Therefore, these transitions are not associated with the formation of $\mathrm{Ar}^{3+}$ and the decay of the remaining $2 p^{-1}$ leads almost exclusively to $\mathrm{Ar}^{3+} \rightarrow \mathrm{Ar}^{4+}$ transitions. The corresponding hypersatellite lines are $2 p^{4} \rightarrow 2 p^{5} 3 p^{4}$ transitions between 215 and $235 \mathrm{eV}$, $2 p^{4} \rightarrow 2 p^{5} 3 s^{1} 3 p^{5}$ transitions between 190 and $198 \mathrm{eV}$, and $2 p^{4} \rightarrow 2 p^{5} 3 s^{0}$ between 172 and $182 \mathrm{eV}$.

The formation of $\mathrm{Ar}^{3+}$ is mostly associated to the second step of the $K L_{2,3} M_{2,3}$ leading to the configuration $2 p^{5} 3 p^{5}$. These transitions are identified in the spectrum as $2 p^{5} 3 p^{5} \rightarrow$ $3 p^{3}$ between 198 and $208 \mathrm{eV}, 2 p^{5} 3 p^{5} \rightarrow 3 s^{1} 3 p^{4}$ between 182 and $190 \mathrm{eV}$, and $2 p^{5} 3 p^{5} \rightarrow 3 s^{0} 3 p^{5}$ between 159 and $164 \mathrm{eV}$.

\section{3. $\mathrm{Ar}^{3+} \rightarrow \mathrm{Ar}^{4+}$ transitions}

The curve regrouping the $\mathrm{Ar}^{3+} \rightarrow \mathrm{Ar}^{4+}$ transitions in Fig. 7 is dominated by a large series of transitions lying in the 139-197 eV kinetic energy region. These transitions are the second step, $2 p^{-1} \rightarrow 2 p^{0}$, of the sequential decay of the $2 p^{-2}$ double vacancy following the $2 p^{-2} \rightarrow 2 p^{-1} L M M$ decay discussed above (noted $L M M 2$ for $\mathrm{HCl}$ in [50]). These transitions are identified as follows: $2 p^{5} 3 p^{4} \rightarrow 3 p^{2}$ between 178 and $197 \mathrm{eV}, 2 p^{5} 3 p^{4} \rightarrow 3 s^{1} 3 p^{3}$ between 155 and $178 \mathrm{eV}$, and $2 p^{5} 3 p^{4} \rightarrow 3 s^{0} 3 p^{4}$ between 139 and $153 \mathrm{eV}$. At higher kinetic energy, we find other minor transitions involving initial states with a $2 s$ hole: $2 s^{1} 3 p^{4} \rightarrow 3 s^{1} 3 p^{4}$ between 230 and $240 \mathrm{eV}, 2 s^{1} 3 p^{4} \rightarrow 3 s^{0} 3 p^{4}$ between 215 and $230 \mathrm{eV}$, and $2 s^{1} 2 p^{5} 3 p^{5} \rightarrow 2 s^{1} 3 p^{3}$ between 200 and $215 \mathrm{eV}$. The latter transition produces a $2 s^{1} 3 p^{3}$ final state that can undergo further Auger decay and lead to the production of $\mathrm{Ar}^{5+}$. All the other transitions have reached a final step in terms of Auger decay and produce $\mathrm{Ar}^{4+}$. As stated above, $K L_{2,3} L_{2,3}$ is the dominant decay channel with a $55.3 \%$ probability. Our 


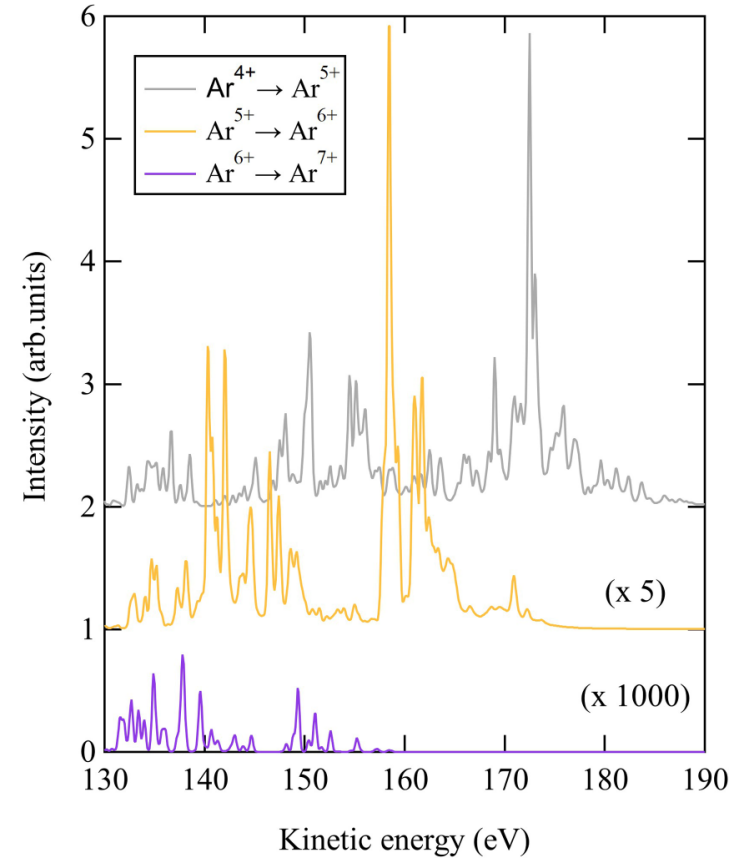

FIG. 8. Partial $\mathrm{Ar}^{4+} \rightarrow \mathrm{Ar}^{5+}, \mathrm{Ar}^{5+} \rightarrow \mathrm{Ar}^{6+}$, and $\mathrm{Ar}^{6+} \rightarrow \mathrm{Ar}^{7+}$ contributions to the $L_{2,3} M M$ Auger decay after core $1 s$ ionization.

tentative disentanglement of the different contributions to the $L_{2,3} M M$ spectrum shows that most transitions eventually lead to the formation of $\mathrm{Ar}^{4+}$, and explains how this ion is the main ion produced after $1 s$ ionization of argon.

\section{4. $\mathrm{Ar}^{4+} \rightarrow \mathrm{Ar}^{5+}, \mathrm{Ar}^{5+} \rightarrow \mathrm{Ar}^{6+}$, and $\mathrm{Ar}^{6+} \rightarrow \mathrm{Ar}^{7+}$ transitions}

So far, we have only considered $L M M$ decay pathways following $K L_{2,3} L_{2,3}$ which represent the majority of all pathways. However, a remaining $29 \%$ is due to first-step $K L_{1} L_{2,3}$ and $K L_{1} L_{1}$ decays. Therefore, there is a possibility that Auger cascades start from $2 s^{-2}$ and $2 s^{-1} 2 p^{-1}$ states, which can end up to higher ionic states. At first these cascades produce electrons in higher kinetic energy region, but subsequent steps can fall into the $L M M$ energy region discussed here. Our theoretical calculations indeed confirm that the low kinetic energy part of the measured $L M M$ spectrum is occupied by weak $\mathrm{Ar}^{4+} \rightarrow \mathrm{Ar}^{5+}, \mathrm{Ar}^{5+} \rightarrow \mathrm{Ar}^{6+}$, and $\mathrm{Ar}^{6+} \rightarrow \mathrm{Ar}^{7+}$ transitions. The partial decay spectra calculated for these transitions are displayed in Fig. 8 and corresponding electronic states are summarized in Table II. All these transitions found between 130 and $190 \mathrm{eV}$ involve an initial state with a single $2 p$ core hole and several $3 s$ and $3 p$ vacancies. Only two very weak groups of transitions were found to reach $\mathrm{Ar}^{7+}$, namely, $2 p^{5} 3 s^{1} 3 p^{2} \rightarrow 3 s^{1} 3 p^{0}$ and $2 p^{5} 3 s^{1} 3 p^{2} \rightarrow 3 s^{0} 3 p^{1}$ between 130 and $160 \mathrm{eV}$.

Our calculations show that the cascade decay pathways followed by the system to reach the highest charge states are both long and complex. To illustrate this, we trace the pathways leading to $\mathrm{Ar}^{7+}$. The main configurations of the six-step Auger decay following $K$-shell ionization can be written as $\left(1 s^{2}\right.$ orbital is not marked for clarity) $2 s^{0} 2 p^{6} 3 s^{2} 3 p^{6} \rightarrow 2 s^{1} 2 p^{5}\left[3 s^{2} 3 p^{5}, 3 s^{1} 3 p^{6}\right] \rightarrow$ $2 s^{2} 2 p^{4}\left[3 s^{2} 3 p^{4}, 3 s^{1} 3 p^{5}\right] \rightarrow 2 s^{1} 2 p^{6}\left[3 s^{2} 3 p^{2}, 3 s^{1} 3 p^{3}, 3 p^{4}\right] \rightarrow$
$2 s^{2} 2 p^{5}\left[3 s^{2} 3 p^{1}, 3 s^{1} 3 p^{2}\right] \rightarrow 2 s^{2} 2 p^{6}\left[3 s^{1}, 3 p^{1}\right]$. In particular, we note the $\mathrm{Ar}^{+4} \rightarrow \mathrm{Ar}^{+5}$ step, where the already filled $2 s$ orbital appears to lose an electron. It can happen because $2 s^{2} 2 p^{4}(3 s 3 p)^{6}$ configurations are indeed higher in energy than $2 s^{1} 2 p^{6}(3 s 3 p)^{4}$ configurations, and because $2 s^{2} 2 p^{4}(3 s 3 p)^{6}$ configurations mix with $2 s^{0} 2 p^{6}(3 s 3 p)^{6}$ and $2 s^{1} 2 p^{5}(3 s 3 p)^{6}$ configurations that provide the nonzero parts to the Auger decay matrix elements. As described above, our calculations only take into account single Auger electron processes. However, transitions where two bound electrons move and one electron is emitted into the continuum, such as $2 s^{2} 2 p^{4}\left[3 s^{2} 3 p^{4}, 3 s^{1} 3 p^{5}\right] \rightarrow 2 s^{1} 2 p^{6}\left[3 s^{2} 3 p^{2}, 3 s^{1} 3 p^{3}, 3 p^{4}\right]$, are included by means of configuration mixing. Indeed, $2 s^{1} 2 p^{6} 3 s^{2} 3 p^{2}$ can mix for instance with $2 s^{2} 2 p^{5} 3 s^{1} 3 p^{3}$, and $2 s^{2} 2 p^{4} 3 s^{2} 3 p^{4} \rightarrow 2 s^{2} 2 p^{5} 3 s^{1} 3 p^{3} \quad$ is a proper nonzero two-electron Auger matrix element. Therefore, $2 s^{2} 2 p^{4}\left[3 s^{2} 3 p^{4}, 3 s^{1} 3 p^{5}\right] \rightarrow 2 s^{1} 2 p^{6}\left[3 s^{2} 3 p^{2}, 3 s^{1} 3 p^{3}, 3 p^{4}\right]$ transition becomes nonzero if $2 s^{2} 2 p^{5} 3 s^{1} 3 p^{3}$ configurations are included to the final state. However, it should be noted that the calculated intensity obtained for $\mathrm{Ar}^{7+}$ in this way is much lower than the experimental intensity. For reasonable agreement, higher-order processes need to be included. For instance, if one includes shakeups as was done recently in atomic cadmium [53], the intensity of $\mathrm{Ar}^{7+}$ would increase.

\section{RESONANT EXCITATION}

Under the same experimental conditions, we have measured the ion mass spectra in coincidence with photons at $3203.5 \mathrm{eV}$, $1 s \rightarrow 4 p$, and $3205.1 \mathrm{eV}, 1 s \rightarrow 5 p$. The measured ion production for each of these resonances is shown in Fig. 9 and compared to the spectrum measured $6 \mathrm{eV}$ above threshold. The first observation is that the $\mathrm{Ar}^{+}$peak dominates the spectrum at the $4 p$ resonance, with a branching ratio of $57.2 \%$, followed by $\mathrm{Ar}^{2+}$ at $35.2 \%$. At the $5 p$ resonance, $\mathrm{Ar}^{+}$and $\mathrm{Ar}^{2+}$ have about the same intensity with branching ratios of $44.6 \%$ and $45.7 \%$, respectively. Table III summarizes the measured and calculated branching ratios for the two resonances compared with the branching ratios obtained above threshold. While a good agreement was found above threshold, our calculations reproduce the experimental findings below threshold only qualitatively. Notably, we find no intensity in the $\mathrm{Ar}^{4+}$ channel while a comparable amount is measured above and below threshold. The reason for completely missing the $\mathrm{Ar}^{4+}$ channel in the below-threshold cases is that even by including $3 d$ and $4 s$ excited correlating configurations, all $\mathrm{Ar}^{+1}$ levels after the first Auger decay are lower in energy than the lowest $\mathrm{Ar}^{4+}$ level. In the above-threshold case, some levels were high enough, bringing some intensity all the way to $\mathrm{Ar}^{+4}$. Including even more configurations would eventually bring some levels high enough also in the below-threshold cases, but the Auger decay rates to these levels would be most likely very small. Therefore, we may conclude that reproducing the experimental results theoretically requires inclusion of double and triple Auger decays in the first step of the cascade.

Compared to the radiative decay above threshold, and in the absence of a photoelectron, $K V_{1,2}$ transitions observed in Fig. 3(a) lead to neutral species. $K L_{1,2}$ decay leads to an excited neutral species with a single hole in the $2 p$ shell and an electron in the $4 p$ or $5 p$ orbital. These highly excited states 

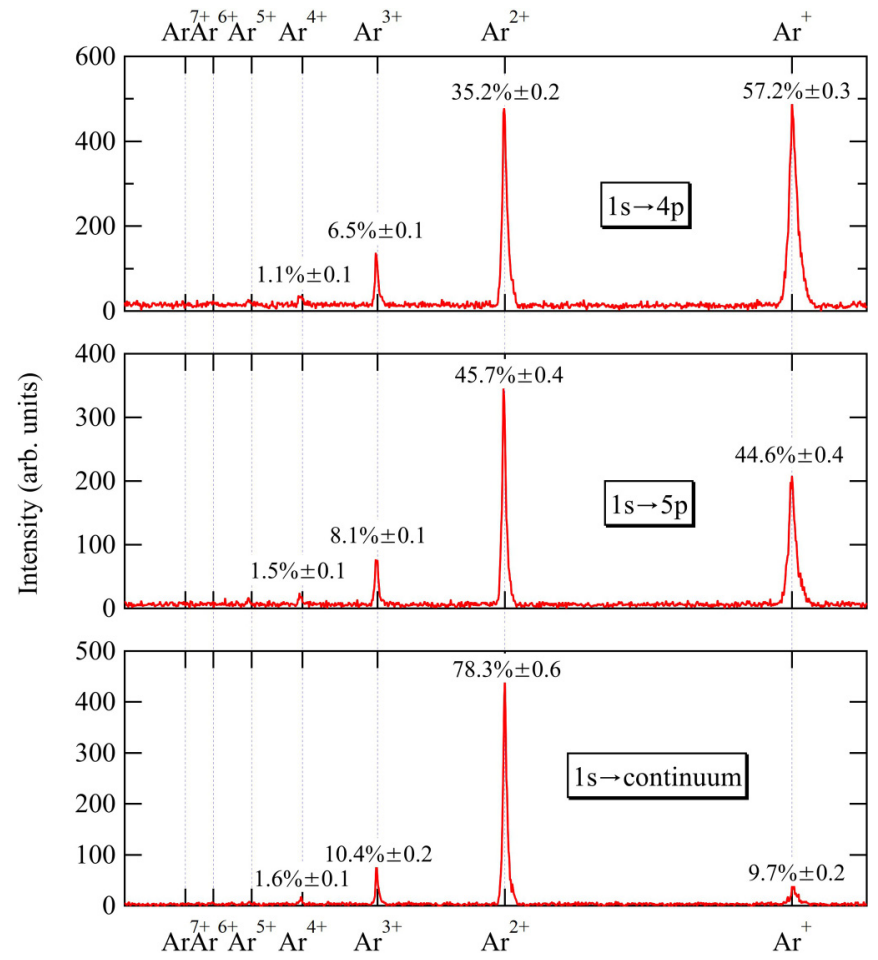

FIG. 9. Comparison of the x-ray photon-ion coincidence spectra measured on top of the $4 p$ resonance (top), $5 p$ resonance (middle), and $6 \mathrm{eV}$ above the ionization threshold (bottom).

further decay via $L M M$ transitions, leading to the formation of a singly charged ion. Similarly to what was stated above for direct ionization, the only electronic states that can further decay to produce $\mathrm{Ar}^{2+}$ are of $3 s^{0} n p^{1}$ configurations. In a simple picture, because $5 p$ electrons interact less with the core vacancies than $4 p$ electrons, the relative probability for spectator decay, not involving the $n p$ excited electron, is higher at the $5 p$ resonance than at the $4 p$ resonance. $3 s^{0} 5 p^{1}$ states are therefore formed more efficiently than $3 s^{0} 4 p^{1}$, and in turn the relative production of $\mathrm{Ar}^{2+}$ is increased at the $5 p$ resonance. Participator decay at the $4 p$ resonance leads to states that cannot further decay, which in turn increases the amount of singly charged ion at the $4 p$ resonance. Although the numbers do not agree with the experimental findings, our calculation results reproduced in Table III predict this tendency.

As in the case of direct photoionization, we have calculated the partial contributions to the $L_{2,3} M M$ Auger decay. The experimental spectrum measured at the $4 p$ resonance is

TABLE III. Experimental and calculated ion branching ratios for photon-ion coincidences for $1 s \rightarrow 4 p$ and $1 s \rightarrow 5 p$ excitations and above-threshold ionization.

\begin{tabular}{|c|c|c|c|c|c|c|}
\hline & \multicolumn{2}{|c|}{$1 s \rightarrow 4 p$} & \multicolumn{2}{|c|}{$1 s \rightarrow 5 p$} & \multicolumn{2}{|c|}{$1 s \rightarrow \epsilon p$} \\
\hline & Expt. & Calc. & Expt. & Calc. & Expt. & Calc. \\
\hline $\mathrm{Ar}^{+}$ & $57.2 \pm 0.3 \%$ & 76.30 & $44.6 \pm 0.4 \%$ & 69.80 & $9.7 \pm 0.2 \%$ & 10.40 \\
\hline $\mathrm{Ar}^{2+}$ & $35.2 \pm 0.2 \%$ & 19.10 & $45.7 \pm 0.4 \%$ & 27.00 & $78.3 \pm 0.6 \%$ & 76.80 \\
\hline $\mathrm{Ar}^{3+}$ & $6.5 \pm 0.1 \%$ & 4.60 & $8.1 \pm 0.1 \%$ & 3.20 & $10.4 \pm 0.2 \%$ & 12.70 \\
\hline $\mathrm{Ar}^{4+}$ & $1.1 \pm 0.1 \%$ & 0 & $1.5 \pm 0.1 \%$ & 0 & $1.6 \pm 0.1 \%$ & 0.12 \\
\hline
\end{tabular}

(a)

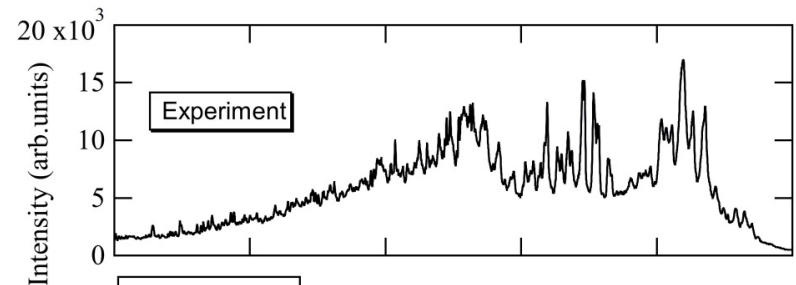

(b)

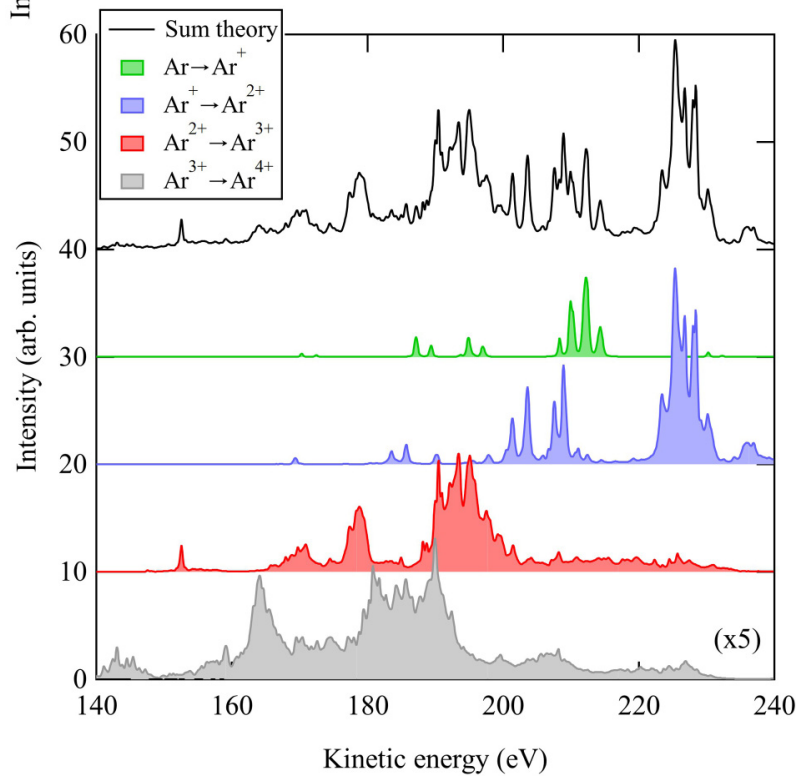

FIG. 10. $L_{2,3} M M$ Auger decay after core $1 s \rightarrow 4 p$ excitation. (a) Experimental spectrum measured on top of the $4 p$ resonance. (b) Total calculated spectrum and partial $\mathrm{Ar}^{n+} \rightarrow \mathrm{Ar}^{m+}$ contributions.

compared to the calculated spectra in Fig. 10. The calculated initial and final states for $\mathrm{Ar}^{n+} \rightarrow \mathrm{Ar}^{m+}$ contributions up to $\mathrm{Ar}^{4+}$ are summarized in Table IV. Transitions to higher ionic states were found to be negligible in the $L_{2,3} M M$ Auger energy range. The general features of the spectrum are fairly well reproduced by the calculations. The main difference is absence of a broad spectrum-wide background that starts to increase from about $140 \mathrm{eV}$ kinetic energy, extending all the way to about $240 \mathrm{eV}$. By comparing Figs. 4 and 10 one can observe that such background is present also in the direct ionization case, but it is considerably more pronounced in the resonant case in Fig. 10. The increase indicates that the background is related to correlation, shakeup, and shakeoff Auger transitions, where the loosely bound $4 p$ electron changes its one-electron orbital during the Auger decay. It is also possible that the $4 p$ electron is shaken off during the first step of the cascade and the subsequent Auger decays fall into the measured energy region. Due to the enormous number of different possibilities for such transitions during the cascade, the spectrum forms a continuous background which overlaps the main spectrum.

De Gouw et al. [54] have studied the resonant Auger decay after $2 p_{3 / 2}$ photoexcitation. However, due to dipole selection rules, photoexcitation below the $2 p$ threshold leads to transitions to the $4 s, 3 d, 4 d$, and $5 d$ unoccupied orbitals, while in our case photoexcitation below the $1 s$ threshold leads to occupancy of the $4 p$ and $5 p$ orbitals. As a result, the measured resonant $L_{2,3} M M$ Auger spectra show different initial and final electronic states. Žitnik et al. [55] have measured the resonant 
TABLE IV. Electronic configurations of initial and final electronic states in the calculated $L_{2,3} M M$ Auger decay after argon $1 s \rightarrow 4 p$ excitation.

\begin{tabular}{|c|c|c|c|}
\hline Transition & $\begin{array}{c}\text { Initial } \\
\text { configuration }\end{array}$ & $\begin{array}{c}\text { Final } \\
\text { configuration }\end{array}$ & $\begin{array}{c}\text { Kinetic } \\
\text { energy }(e V)\end{array}$ \\
\hline \multirow[t]{4}{*}{$\mathrm{Ar}^{0} \rightarrow \mathrm{Ar}^{+}$} & $2 p^{5} 4 p^{1}$ & $3 p^{5}$ & $225-235$ \\
\hline & $2 p^{5} 4 p^{1}$ & $3 p^{4} 4 p^{1}$ & $203-220$ \\
\hline & $2 p^{5} 4 p^{1}$ & $3 s^{1} 3 p^{5} 4 p^{1}$ & $180-200$ \\
\hline & $2 p^{5} 4 p^{1}$ & $3 s^{0} 4 p^{1}$ & $167-174$ \\
\hline \multirow[t]{6}{*}{$\mathrm{Ar}^{+} \rightarrow \mathrm{Ar}^{2+}$} & $2 p^{4} 4 p^{1}$ & $2 p^{5} 3 p^{4} 4 p^{1}$ & $215.5-240$ \\
\hline & $2 p^{5} 3 p^{5} 4 p^{1}$ & $3 p^{3} 4 p^{1}$ & $205.3-215.5$ \\
\hline & $2 p^{4} 4 p^{1}$ & $2 p^{5} 3 s^{1} 3 p^{5} 4 p^{1}$ & 199-205.3 \\
\hline & $2 p^{5} 3 p^{5} 4 p^{1}$ & $3 s^{1} 3 p^{4} 4 p^{1}$ & 188-199 \\
\hline & $2 p^{4} 4 p^{1}$ & $2 p^{5} 3 s^{0} 4 p^{1}$ & $180-188$ \\
\hline & $2 p^{5} 3 p^{5} p 4 p^{1}$ & $3 s^{0} 3 p^{5} 4 p^{1}$ & $160-173$ \\
\hline \multirow[t]{6}{*}{$\mathrm{Ar}^{2+} \rightarrow \mathrm{Ar}^{3+}$} & $2 p^{5} 3 p^{4} 4 p^{1}$ & $3 p^{3}$ & $220-235$ \\
\hline & $2 p^{4} 3 p^{5} 4 p^{1}$ & $2 p^{5} 3 s^{1} 3 p^{4} 4 p^{1}$ & $209.5-225$ \\
\hline & $2 p^{4} 3 s^{1} 4 p^{1}$ & $2 p^{5} 3 s^{1} 3 p^{4} 4 p^{1}$ & $202.5-219$ \\
\hline & $2 p^{5} 3 p^{4} 4 p^{1}$ & $3 p^{2} 4 p^{1}$ & $186-202.5$ \\
\hline & $2 p^{5} 3 p^{4} 4 p^{1}$ & $3 s^{1} 3 p^{3} 4 p^{1}$ & $162-186$ \\
\hline & $2 p^{5} 3 p^{4} 4 p^{1}$ & $3 s^{0} 3 p^{4} 4 p^{1}$ & $145-160$ \\
\hline \multirow[t]{9}{*}{$\mathrm{Ar}^{3+} \rightarrow \mathrm{Ar}^{4+}$} & $2 p^{5} 3 s^{0} 3 p^{5} 4 p^{1}$ & $3 s^{0} 3 p^{4}$ & $203-235$ \\
\hline & $2 p^{5} 3 s^{1} 3 p^{4} 4 p^{1}$ & $3 s^{1} 3 p^{3}$ & \\
\hline & $2 p^{5} 3 p^{3} 4 p^{1}$ & $3 p^{2}$ & \\
\hline & $2 p^{5} 3 s^{0} 3 p^{5} 4 p^{1}$ & $3 p^{3} 4 p^{1}$ & 185-196 \\
\hline & $2 p^{5} 3 s^{1} 3 p^{4} 4 p^{1}$ & $3 s^{1} 3 p^{2} 4 p^{1}$ & $183.5-187$ \\
\hline & $2 p^{5} 3 p^{3} 4 p^{1}$ & $3 p^{1} 4 p^{1}$ & $178.3-183.2$ \\
\hline & $2 p^{5} 3 s^{1} 3 p^{4} 4 p^{1}$ & $3 s^{0} 3 p^{3} 4 p^{1}$ & $150-173.8$ \\
\hline & $2 p^{5} 3 p^{3} 4 p^{1}$ & $3 s^{1} 3 p^{2} 4 p^{1}$ & \\
\hline & $2 p^{5} 3 p^{3} 4 p^{1}$ & $3 s^{0} 3 p^{3} 4 p^{1}$ & $134-150$ \\
\hline
\end{tabular}

Auger spectra after $2 p$ excitation by electron impact, where excitation to the $4 p$ and $5 p$ orbitals is possible. However, the energy resolution obtained with this technique did not allow a clear separation of the different contributions.

In our measurements, spectra and electronic states obtained for the $1 s \rightarrow 5 p$ excitation were found to be very similar and are not shown in this article. Compared to the results obtained above ionization threshold, no fundamental differences in the electronic configurations are found, apart from the presence of a spectator electron in the $4 p$ or $5 p$ orbital. It shows, however, that at this stage of the cascade, the decay is almost exclusively spectator decay and a large majority of the final states still have an electron in $4 p$ or $5 p$ orbital. Only weak transitions to final states with $3 p^{3}$ configurations are found in the $\mathrm{Ar}^{2+} \rightarrow \mathrm{Ar}^{3+}$ partial Auger spectrum and with $3 s^{0} 3 p^{4}$ and $3 p^{2}$ configurations in $\mathrm{Ar}^{3+} \rightarrow \mathrm{Ar}^{4+}$.

\section{CONCLUSION}

Taking advantage of a unique combination of synchrotron radiation-based experimental techniques, we have investigated the main relaxation channels involved in the formation of highly charged ions after core $1 s$ ionization of argon. The complementarity of the several different spectroscopic methods we used leads to a better understanding of the fine details of the complex decay pathways involving radiative and nonradiative relaxation after deep core-level ionization. Theoretical calculations were carried out to explain the experimental findings. It was shown that the present-day models are capable of reproducing complex electron and ion spectra arising from up to six-step Auger cascades with fairly good accuracy.

\section{ACKNOWLEDGMENTS}

Experiments were performed on the GALAXIES and LUCIA beamlines at SOLEIL Synchrotron, France (Proposal No. 20120122). We are grateful to D. Prieur for technical assistance and to SOLEIL staff for smoothly running the facility.
[1] T. A. Carlson and M. O. Krause, Phys. Rev. 137, A1655 (1965).

[2] A. G. Kochur, V. L. Sukhorukov, A. I. Dudenko, and Ph. V. Demekhin, J. Phys. B: At., Mol. Opt. Phys. 28, 387 (1995).

[3] T. LeBrun, S. H. Southworth, G. B. Armen, M. A. MacDonald, and Y. Azuma, Phys. Rev. A 60, 4667 (1999).

[4] W. Wölfli, C. Stoller, G. Bonani, M. Suter, and M. Stöckli, Phys. Rev. Lett. 35, 656 (1975).

[5] M. Žitnik, R. Püttner, G. Goldsztejn, K. Bučar, M. Kavčič, A. Mihelič, T. Marchenko, R. Guillemin, L. Journel, O. Travnikova, D. Céolin, M. N. Piancastelli, and M. Simon, Phys. Rev. A 93, 021401(R) (2016).

[6] A. Cesar and H. Ågren, Phys. Rev. A 45, 2833 (1992).

[7] J.-E. Rubensson, M. Neeb, A. Bringer, M. Biermann, and W. Eberhardt, Chem. Phys. Lett. 257, 447 (1996).

[8] D. Céolin, T. Marchenko, R. Guillemin, L. Journel, R. K. Kushawaha, S. Carniato, S.-M. Huttula, J.-P. Rueff, G. B. Armen, M. N. Piancastelli, and M. Simon, Phys. Rev. A 91, 022502 (2015).
[9] R. K. Kushawaha, K. Jänkälä, T. Marchenko, G. Goldsztejn, R. Guillemin, L. Journel, D. Céolin, J.-P. Rueff, A. F. Lago, R. Püttner, M. N. Piancastelli, and M. Simon, Phys. Rev. A 92, 013427 (2015).

[10] G. Goldsztejn, T. Marchenko, D. Céolin, L. Journel, R. Guillemin, J.-P. Rueff, R. K. Kushawaha, R. Püttner, M. N. Piancastelli, and M. Simon, Phys. Chem. Chem. Phys. 18, 15133 (2016).

[11] G. Goldsztejn, R. Püttner, L. Journel, R. Guillemin, O. Travnikova, R. K. Kushawaha, B. Cunha de Miranda, I. Ismail, D. Céolin, M. N. Piancastelli, M. Simon, and T. Marchenko, Phys. Rev. A 95, 012509 (2017).

[12] M. O. Krause, J. Phys. Chem. Ref. Data 8, 307 (1979).

[13] U. Alkemper, J. Doppelfeld, and F. von Busch, Phys. Rev. A 56, 2741 (1997).

[14] F. von Busch, U. Kuetgens, J. Doppelfeld, and S. Fritzsche, Phys. Rev. A 59, 2030 (1999). 
[15] K. Ueda, E. Shigemasa, Y. Sato, A. Yagishita, M. Ukai, H. Maezawa, T. Hayaishi, and T. Sasaki, J. Phys. B: At., Mol. Opt. Phys. 24, 605 (1991).

[16] D. V. Morgan, R. J. Bartlett, and M. Sagurton, Phys. Rev. A 51, 2939 (1995).

[17] R. Guillemin, C. Bomme, T. Marin, L. Journel, T. Marchenko, R. K. Kushawaha, N. Trcera, M. N. Piancastelli, and M. Simon, Phys. Rev. A 84, 063425 (2011).

[18] U. Arp, J. W. Cooper, T. LeBrun, S. H. Southworth, M. Jung, and M. A. MacDonald, J. Phys. B: At., Mol. Opt. Phys. 29, L837 (1996).

[19] U. Arp, T. LeBrun, S. H. Southworth, M. A. MacDonald, and M. Jung, Phys. Rev. A 55, 4273 (1997).

[20] A. Kahoul, A. Abassi, B. Deghfel, and M. Nekkab, Radiat. Phys. Chem. 80, 369 (2011), and references therein.

[21] R. Guillemin, S. Carniato, L. Journel, W. C. Stolte, T. Marchenko, L. El Khoury, E. Kawerk, M. N. Piancastelli, A. Hudson, D. W. Lindle, and M. Simon, J. Electron Spectrosc. Relat. Phenom. 188, 53 (2013).

[22] J. C. Levin, C. Biedermann, N. Keller, L. Liljeby, C.-S. O, R. T. Short, I. A. Sellin, and D. W. Lindle, Phys. Rev. Lett. 65, 988 (1990).

[23] D. Vantelon, N. Trcera, D. Roy, T. Moreno, D. Mailly, S. Guilet, E. Metchalkov, F. Delmotte, B. Lassalle, P. Lagarde, and A.-M. Flank, J. Synchrotron Radiat. 23, 635 (2016).

[24] J.-P. Rueff, J. M. Ablett, D. Céolin, D. Prieur, Th. Moreno, V. Balédent, B. Lassalle-Kaiser, J. E. Rault, M. Simon, and A. Shukla, J. Synchrotron Radiat. 22, 175 (2015).

[25] C. Bomme, R. Guillemin, T. Marin, L. Journel, T. Marchenko, D. Dowek, N. Trcera, B. Pilette, A. Avila, H. Ringuenet, R. K. Kushawaha, and M. Simon, Rev. Sci. Instrum. 84, 103104 (2013).

[26] R. Guillemin, S. Sheinerman, C. Bomme, L. Journel, T. Marin, T. Marchenko, R. K. Kushawaha, N. Trcera, M. N. Piancastelli, and M. Simon, Phys. Rev. Lett. 109, 013001 (2012).

[27] C. Bomme, R. Guillemin, S. Sheinerman, T. Morin, L. Journel, T. Marchenko, R. K. Kushawaha, N. Trcera, M. N. Piancastelli, and M. Simon, J. Phys. B: At., Mol. Opt. Phys. 46, 215101 (2013).

[28] C. Bomme, R. Guillemin, T. Marin, L. Journel, T. Marchenko, N. Trcera, R. K. Kushawaha, M. N. Piancastelli, M. Simon, M. Stener, and P. Decleva, J. Phys. B: At., Mol. Opt. Phys. 45, 194005 (2012).

[29] R. Guillemin, P. Decleva, M. Stener, C. Bomme, T. Marin, L. Journel, T. Marchenko, R. K. Kushawaha, K. Jänkälä, N. Trcera, K. P. Bowen, D. W. Lindle, M. N. Piancastelli, and M. Simon, Nat. Commun. 6, 6166 (2015).

[30] R. Guillemin, S. Sheinerman, R. Püttner, T. Marchenko, G. Goldsztejn, L. Journel, R. K. Kushawaha, D. Céolin, M. N. Piancastelli, and M. Simon, Phys. Rev. A 92, 012503 (2015).

[31] D. Céolin, J. M. Ablett, D. Prieur, T. Moreno, J.-P. Rueff, T. Marchenko, L. Journel, R. Guillemin, B. Pilette, T. Marin, and M. Simon, J. Electron Spectrosc. Relat. Phenom. 190, 188 (2013).

[32] M. Simon, R. Püttner, T. Marchenko, R. Guillemin, R. K. Kushawaha, L. Journel, G. Goldsztejn, M. N. Piancastelli, J. M. Ablett, J.-P. Rueff, and D. Céolin, Nat. Commun. 5, 4069 (2014).
[33] R. Püttner, G. Goldsztejn, D. Céolin, J.-P. Rueff, T. Moreno, R. K. Kushawaha, T. Marchenko, R. Guillemin, L. Journel, D. W. Lindle, M. N. Piancastelli, and M. Simon, Phys. Rev. Lett. 114, 093001 (2015).

[34] G. Goldsztejn, T. Marchenko, R. Püttner, L. Journel, R. Guillemin, S. Carniato, P. Selles, O. Travnikova, D. Céolin, A. F. Lago, R. Feifel, P. Lablanquie, M. N. Piancastelli, F. Penent, and M. Simon, Phys. Rev. Lett. 117, 133001 (2016).

[35] O. Travnikova, N. Sisourat, T. Marchenko, G. Goldsztejn, R. Guillemin, L. Journel, D. Céolin, I. Ismail, A. F. Lago, R. Püttner, M. N. Piancastelli, and M. Simon, Phys. Rev. Lett. 118, 213001 (2017)

[36] P. Jönsson, X. He, C. Froese Fischer, and I. P. Grant, Comput. Phys. Commun. 177, 597 (2007).

[37] P. Jönsson, G. Gaigalas, J. Bieroń, C. Froese Fischer, and I. P. Grant, Comput. Phys. Commun. 184, 2197 (2013).

[38] S. Fritzsche, C. Froese Fischer, and G. Gaigalas, Comput. Phys. Commun. 148, 103 (2002).

[39] S. Fritzsche, Comput. Phys. Commun. 183, 1525 (2012).

[40] S. Fritzsche, C. Froese Fischer, and C. Z. Dong, Comput. Phys. Commun. 124, 340 (2000).

[41] M. H. Chen, B. Crasemann, and H. Mark, Phys. Rev. A 24, 177 (1981).

[42] T. Hayaishi, E. Murakami, A. Yagishita, F. Koike, Y. Morioka, and J. E. Hansen, J. Phys. B: At., Mol. Opt. Phys. 21, 3203 (1988).

[43] N. Saito and I. H. Suzuki, J. Phys. Soc. Jpn. 66, 1979 (1997).

[44] S. Brünken, Ch. Gerth, B. Kanngießer, T. Luhmann, M. Richter, and P. Zimmermann, Phys. Rev. A 65, 042708 (2002).

[45] T. A. Carlson and M. O. Krause, Phys. Rev. Lett. 17, 1079 (1966).

[46] J. Viefhaus, S. Cvejanovíc, B. Langer, T. Lischke, G. Prümper, D. Rolles, A. V. Golovin, A. N. Grum-Grzhimailo, N. M. Kabachnik, and U. Becker, Phys. Rev. Lett. 92, 083001 (2004).

[47] P. Lablanquie, L. Andric, J. Palaudoux, U. Becker, M. Braune, J. Viefhaus, J. H. D. Eland, and F. Penant, J. Electron Spectrosc. Relat. Phenom. 156-158, 51 (2007).

[48] P. Lablanquie, M. A. Khalal, L. Andric, J. Palaudoux, F. Penent, J.-M. Bizau, D. Cubaynes, K. Jänkälä, Y. Hikosaka, K. Ito, K. Bučar, and M. Žitnik, J. Electron Spectrosc. Relat. Phenom. 220, 125 (2017).

[49] M. H. Chen, B. Crasemann, and H. Mark, At. Data Nucl. Data Tables 24, 13 (1979).

[50] O. Travnikova, T. Marchenko, G. Goldsztejn, K. Jänkälä, N. Sisourat, S. Carniato, R. Guillemin, L. Journel, D. Céolin, R. Püttner, H. Iwayama, E. Shigemasa, M. N. Piancastelli, and M. Simon, Phys. Rev. Lett. 116, 213001 (2016).

[51] H. Pulkkinen, S. Aksela, O.-P. Sairanen, A. Hiltunen, and H. Aksela, J. Phys. B: At., Mol. Opt. Phys. 29, 3033 (1996).

[52] J. A. R. Samson, Y. Lu, and W. C. Stolte, Phys. Rev. A 56, R2530(R) (1997).

[53] R. Beerwerth and S. Fritzsche, Eur. Phys. J. D 71, 253 (2017).

[54] J. A. de Gouw, J. van Eck, A. C. Peters, J. van der Weg, and H. G. M. Heideman, J. Phys. B: At., Mol. Opt. Phys. 28, 2127 (1995).

[55] M. Žitnik, M. Kavčič, K. Bučar, B. Paripás, B. Palásthy, and K. Tőkési, Nucl. Instrum. Methods Phys. Res., Sect. B 267, 260 (2009). 